\title{
Aneurisma de veia poplítea: relato de caso e revisão da literatura
}

\section{Popliteal vein aneurysm: case report and literature review}

\author{
Bruno Morisson'1, Jose Marcos Braz Serafim', Edwal Balthazar dos Santos², Davi Pinto Colhen³, Bruno Miana Caiafa ${ }^{3}$, \\ Ronaldo Carvalho ${ }^{3}$
}

\begin{abstract}
Resumo
Os aneurismas venosos são raros, porém causam complicações tromboembólicas. Na maioria das vezes, são encontrados como achados de exame físico ou de imagem. Os aneurismas sintomáticos de veia poplítea são tratados por reparo cirúrgico, devido ao alto risco de recorrência de embolia pulmonar. A técnica mais utilizada é a aneurismectomia tangencial com venorrafia lateral. Na impossibilidade de se empregar esta técnica, faz-se a ressecção com reconstrução venosa. Os autores relatam o caso de uma paciente com aneurisma de veia poplítea, cujo diâmetro era de 44 mm, submetida à aneurismectomia tangencial e venorrafia lateral, com sucesso.
\end{abstract}

Palavras-chave: Aneurisma; veia; cirurgia

\begin{abstract}
Venous aneurysms are rare; however, they are potential causes of thromboembolism. Most cases are found as clinical examination or by imaging methods. Symptomatic aneurysms of the popliteal vein are treated by surgical repair due to the high risk of recurrent pulmonary embolism. The most widely used procedure is tangential aneurysmectomy and lateral venorrhaphy. If this technique is not possible, the aneurysm should be removed and venous reconstruction should be performed. The authors report the case of a patient with popliteal vein aneurysm measuring $44 \mathrm{~mm}$ in diameter which was successfully treated by tangential aneurysmectomy and lateral venorrhaphy.
\end{abstract}

Keywords: Aneurysm; vein; surgery

\section{Introdução}

O termo aneurisma é utilizado para caracterizar uma dilatação dos vasos sanguíneos. É relacionado às dilatações arteriais; entretanto, essas lesões podem ocorrer em qualquer parte do sistema vascular.

Aneurismas venosos são pouco frequentes e geralmente aparecem como achados. Esses raramente têm significância clínica, com exceção dos aneurismas de veia poplítea (AVP), que devem ser lembrados como uma rara causa de embolia pulmonar recorrente e de trombose venosa profunda ${ }^{1}$.

A maioria dos aneurismas venosos é de origem congênita, podendo ser adquiridos por trauma, processos inflamatórios e alterações degenerativas.

\section{Relato de caso}

Trata-se de paciente, sexo feminino, cor negra, com 21 anos de idade, queixando-se de dor, cansaço, edema e sensação de peso na perna esquerda, predominantemente na fossa poplítea. Não possui antecedentes de trombose venosa profunda e relata dispneia ocasional aos grandes esforços.

Ao exame físico, constatou-se a presença de pequeno abaulamento na fossa poplítea esquerda quando em posição ortostática, que desaparecia com a elevação do membro.

O exame eco-Doppler evidenciou refluxo no sistema venoso profundo, no membro inferior esquerdo; dilatação fusiforme da veia poplítea (Figura 1), com diâmetros

Trabalho realizado no Hospital Federal do Andaraí (HFA) - Andaraí (RJ), Brasil. 
transversos de 44 x $35 \mathrm{~mm}$. Realizou-se também flebografia ascendente, que confirmou os achados (Figura 2).

A paciente foi preparada para reparo cirúrgico com préoperatório constando: hemograma, bioquímica, eletrocardiograma, radiografia de tórax e ecocardiograma normais, caracterizando risco cirúrgico tipo I conforme a escala ASA.

Foi submetida à aneurismectomia tangencial com venorrafia lateral (Figuras 3, 4, 5 e 6), por abordagem poplítea posterior.

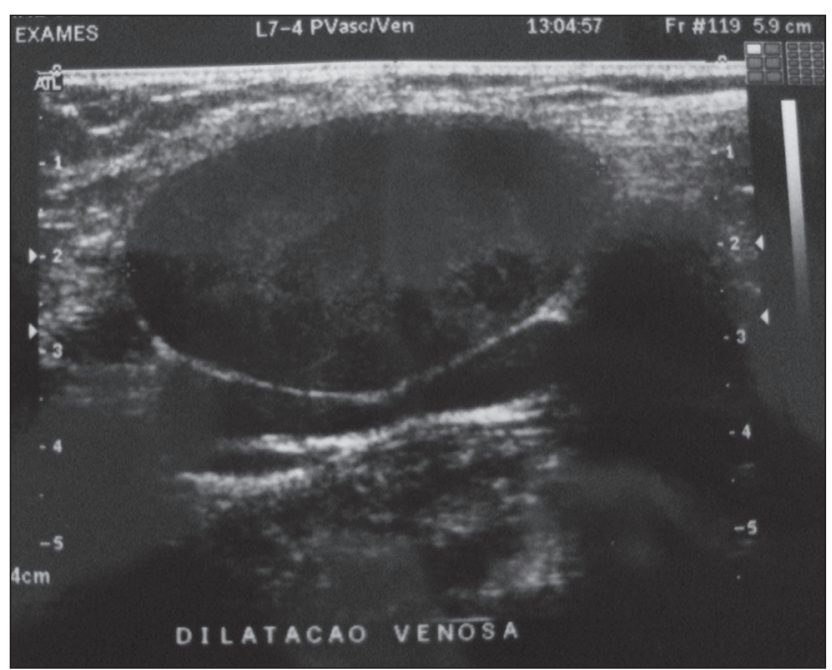

Figura 1 - Doppler

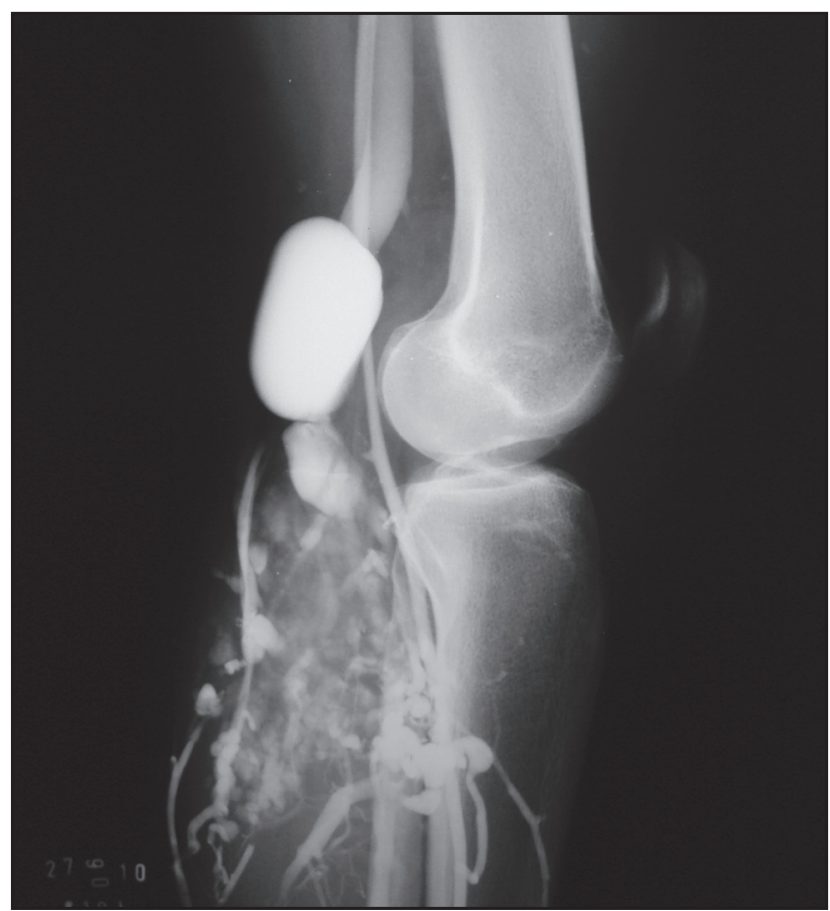

Figura 2 - Flebografia

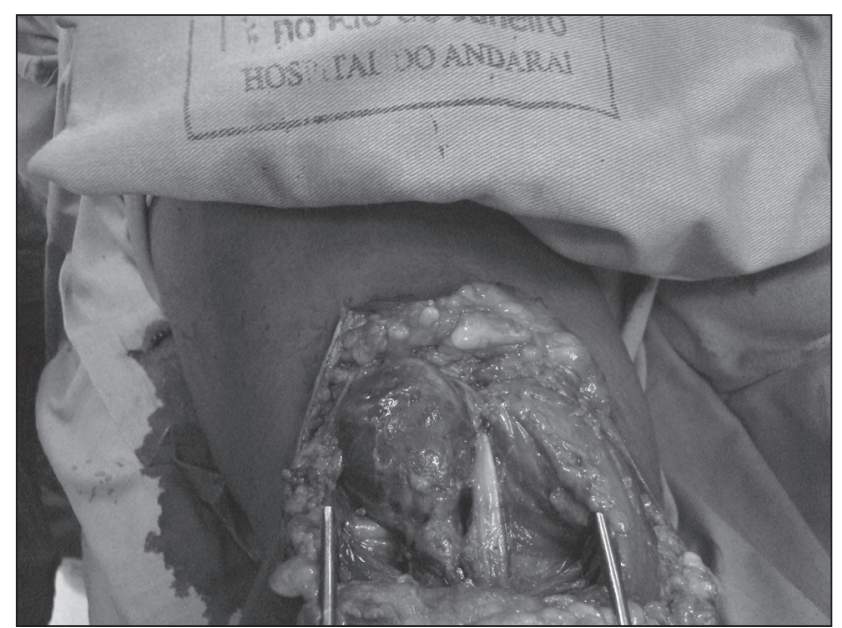

Figura 3 - Dissecção do aneurisma

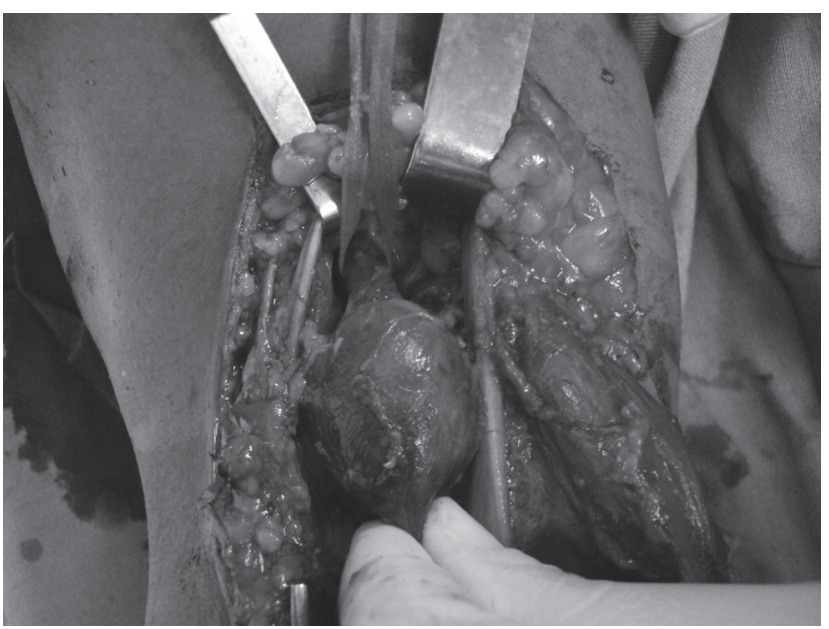

Figura 4 - Dissecção do aneurisma

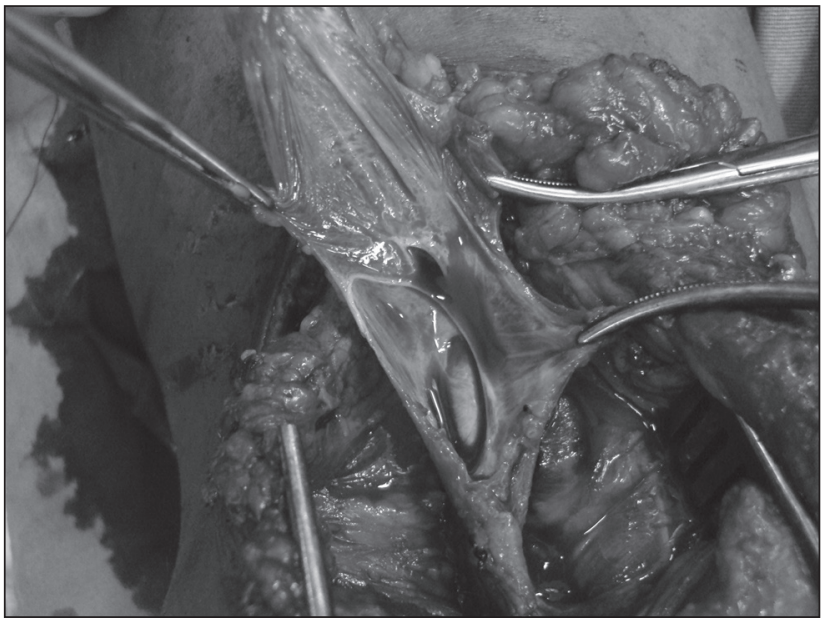

Figura 5 - Aneurismectomia tangencial 


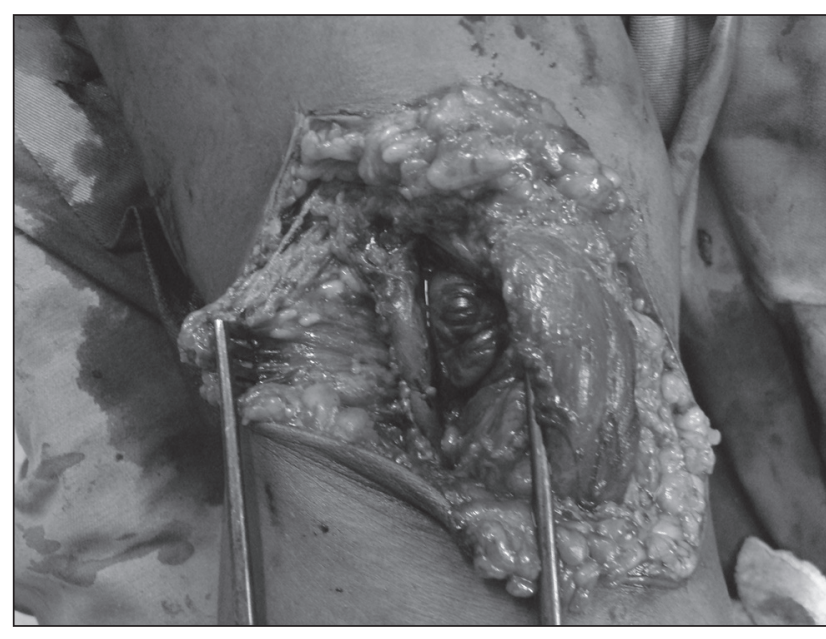

Figura 6 - Venorrafia lateral

No terceiro dia de pós-operatório, a paciente evoluiu apresentando hematoma volumoso em fossa poplítea com edema e dor importante no membro. Foi realizado eco-Doppler colorido que não visualizou veia poplítea, porém foram visualizadas veias com fluxo, sem trombose a jusante $\mathrm{e}$ a montante o sítio cirúrgico, concluiu-se que era um hematoma comprimindo a veia rafiada, optou-se por drenagem do mesmo, com melhora substancial da dor e do edema.

A paciente recebeu alta 14 dias após o procedimento, sem edemas ou dores com prescrição de anticoagulantes (varfarina) e flebotônicos (diosmina com hisperidina) por via oral, durante 90 dias.

Houve melhora dos sintomas relatados previamente, estando a paciente em acompanhamento ambulatorial sem a prescrição de cumarínicos, mantendo apenas o flebotônico com elastocompressão.

\section{Discussão}

A primeira publicação sobre aneurisma venoso foi feita por Osler em 1915, o qual relatou um caso de dilatação da veia axilar. Posteriormente, em 1928, Harris, descreveu uma criança de cinco anos com aneurisma de veia jugular ${ }^{2,3}$.

Abbott descreveu um caso de aneurisma de veia cava superior em 1950. Em 1968, May e Nissel foram os primeiros a descreverem o AVP, e Dahl et al., os primeiros a relatarem as complicações dos $\mathrm{AVP}^{3}$.

Não há um critério preciso quanto ao diâmetro do vaso para definir uma dilatação venosa como aneurismática, foi proposto que tal definição seria correta diante de uma dilatação duas vezes maior do que o diâmetro normal do vaso.
Alguns autores não concordaram com esta definição e afirmam a necessidade desse diâmetro ser três vezes maior do que aquele da veia poplítea normal para tal consideração ${ }^{3}$.

Diferentemente do sistema arterial, aneurismas do sistema venoso são raros e ocorrem em qualquer idade, sem distinção de sexo. Não há concordância na literatura a respeito da localização mais frequente dos aneurismas venosos. Dourado et al. ${ }^{2}$ descreveram dois casos de aneurismas venosos no nível do pé.

A perda focal de componentes do tecido conjuntivo normal da parede da veia, devido a uma falha congênita de desenvolvimento ou perdas degenerativas associadas à idade, ou ainda ao processo inflamatório, resulta no enfraquecimento da parede, tornando-a vulnerável à dilatação.

Devido às alterações estruturais, os aneurismas venosos se apresentam de duas formas: fusiformes ou saculares. Essa diferenciação é importante para determinar a estratégia cirúrgica.

Os AVP saculares representam $75 \%$ dos casos ${ }^{3}$. Uma ampla variedade de apresentações clínicas dos aneurismas venosos é descrita na literatura. A maioria dos casos de aneurismas venosos periféricos causa apenas desconforto local, sendo, portanto, assintomática, com diagnóstico incidental. No caso apresentado, a paciente manifestou sintomas dolorosos no membro inferior esquerdo. No entanto, essa entidade pode se manifestar por complicações, como trombose venosa profunda e embolia pulmonar recorrente.

Os aneurismas venosos periféricos podem ser detectados pela presença de massa subcutânea macia, que desaparece com a elevação do membro e aumenta com a manobra de Valsalva. A suspeita será confirmada por ultrassonografia vascular, método de escolha para o diagnóstico definitivo; a flebografia ascendente e a ressonância nuclear magnética ficam restritas aos casos de aneurismas venosos abdominais ou torácicos. Entretanto, optou-se pela flebografia para a determinação precisa da anatomia venosa antes da cirurgia.

As complicações mais comuns dos aneurismas venosos são: a trombose venosa profunda, a tromboflebite e a embolia pulmonar recorrente.

A cirurgia é o tratamento dos AVP. Em pacientes que apresentam complicações tromboembólicas, o tratamento cirúrgico é mandatório e considerado como de escolha, limitado apenas pelas condições clínicas, visto que a anticoagulação isolada nos pacientes com embolia pulmonar é ineficaz, com altos índices de recorrência $(80 \%)^{3}$. A indicação de tratamento cirúrgico em pacientes com AVP assintomáticos é contraditória. Entretanto, aneurismas saculares de qualquer tamanho ou grandes aneurismas fusiformes deveriam 
ser tratados cirurgicamente, devido ao potencial de eventos tromboembólicos futuros. A recorrência de embolia pulmonar após a cirurgia nunca foi descrita. Os AVP fusiformes pequenos e assintomáticos podem ser acompanhados clinicamente com estudos periódicos de eco-Doppler.

A aneurismectomia tangencial com venorrafia lateral é o método cirúrgico mais utilizado e tem sido recomendada para aneurismas saculares ${ }^{3}$, mas também pode ser realizada em aneurismas fusiformes, como no presente caso. A ressecção do aneurisma e a reconstrução venosa são recomendadas $^{4}$ quando a aneurismectomia tangencial não pode ser realizada, devido ao risco de permanência de segmento da doença na parede venosa.

Várias opções cirúrgicas são passíveis de realização, tais como a ressecção do aneurisma com anastomose terminoterminal, ressecção com interposição de enxerto venoso autógeno homólogo, prótese de politetrafluoretileno (PTFE), bypass venoso, ressecção com transposição venosa (tronco tibioperoneal para veia tibial anterior, veia do gastrocnêmio medial) e ressecção sem reconstrução venosa e ligadura. Deve ser citado, ainda, o implante de filtro de veia cava inferior ${ }^{4}$.

Embora a veia safena magna ipsilateral possa ser utilizada como substituta, esta estratégia deve ser evitada pelo risco da perda de uma importante via colateral caso ocorra trombose após o reparo cirúrgico.

A perviedade da reconstrução da veia poplítea é pouco documentada na literatura. Os resultados da ressecção com anastomose termino-terminal não são conhecidos. $\mathrm{O}$ resultado no longo prazo parece ser satisfatório para a reconstrução com remendo venoso ou enxerto interposto de safena, diferente de quando são utilizados enxertos sintéticos e de veia jugular interna.

\section{Conclusão}

O AVP é uma ocorrência rara, que pode causar sérias complicações tromboembólicas. Essa afecção deve ser considerada em pacientes com episódios de embolia pulmonar de repetição e ausência de fatores de risco para tromboembolismo.

O tratamento cirúrgico é indicado para todos os pacientes com AVP sintomático, devido ao risco elevado da recorrência de embolia pulmonar. Os AVP assintomáticos, porém grandes ou saculares, podem também ser tratados cirurgicamente.

O tratamento mais utilizado é a aneurismectomia tangencial com venorrafia lateral. Entretanto, quando essa técnica não pode ser utilizada, deve-se fazer a ressecção com reconstrução. Os pequenos aneurismas, como os menores de $20 \mathrm{~mm}$ e sem trombos no seu interior, podem ser acompanhados com eco-Doppler seriado.

\section{Referências}

1. Herrera LJ, Davis JW, Livesay JJ. Popliteal Vein Aneurysm Presenting as a Popliteal Mass. Tex Heart Inst J. 2006;33(2):246-8.

2. Dourado OC, Miranda AG, Filho AP, Dourado LO. Aneurisma Venoso no Pé: Relato de caso e revisão da literatura. J Vasc Bras. 2006;5(4):313-6.

3. Thomazinho F, Diniz JAM, Junior RAEH, Diniz CAM, Perozin IS. Aneurisma de veia poplítea: relato de caso e revisão de literatura. J Vasc Bras. 2008;7(3):6-7.

4. Gillespie DL, Villavicencio JL, Gallagher C, et al. Presentation and management of venous aneurysms. J Vasc Surg. 1997;26:845-52.

Correspondência :
Bruno Morisson
Rua Leopoldo, 280 - Andaraí CEP: 20541-170
Rio de Janeiro (RJ), Brasil
Tel.: (21) 2575-7033
E-mail: morisson@terra.com.br
Contribuições dos autores:
Concepção e desenho do estudo: BM
Análise e interpretação dos dados: RC
Coleta de dados: BMC
Redação do artigo: BM
Revisão crítica do texto: EBS
Aprovação final do artigo*: JMBS
Análise estatística: DPC
Responsabilidade geral pelo estudo: BM
*Todos os autores leram e aprovaram a versão final submetida ao J Vasc Bras.

